Ann. Sci. forest., 1980, 37 (1), 73-83.

\title{
Influence des enveloppes séminales sur l'imbibition des glands de chêne pédonculé (Q. robur L.)
}

\author{
M. LAMOND ef J. LEVERT \\ Laboratoire de Phytomorphogenèse $\mathrm{n}^{\circ} 45$ ASS. au CNRS \\ 4, rue Ledru, 63000 Clermont-Ferrand.
}

\begin{abstract}
Résumé
En germoir, l'imbibition des glands de chêne pédonculé s'effectue de façon graduelle et reste très faible jusqu'à la germination. Les embryons de ces semences se réhydratent par contre fortement dès qu'ils sont isolés et placés en contact avec le substrat humide.

Des expériences d'immersion de semences (embryons nus, glands intacts, glands à hile scellé à la paraffine) dans l'eau courante, ont montré : 1. - que le hile est la zone la plus perméable du péricarpe ; 2. - que les embryons ne se réhydratent presque pas à l'intérieur de leurs enveloppes même lorsque la teneur en eau de ces dernières augmente fortement.

La faible perméabilité des secteurs du péricarpe généralement en contact avec le substrat humide ainsi que la reprise d'eau négligeable des embryons à l'intérieur de leurs enveloppes expliquent, en grande partie, la mauvaise germination de glands dont la teneur en eau est relativement faible ( 30 à 35 p. 100 mat. fraîche).
\end{abstract}

\section{Introduction}

Les glands, comme la plupart des grosses semences d'essences forestières à teneur en eau relativement élevée, sont difficiles à conserver. Ils germent rapidement même à basse température s'ils sont entreposés sans ressuyage préalable (Camus, 1952 ; Bonner, 1971 ; Wang, 1974 ; Claudot, 1974 ; Bonnet-Masimbert ef al., 1977) ; ils germent très mal et peuvent même perdre tout pouvoir germinatif lorsqu'ils sont trop desséchés (Watt, 1919 ; Bonner, 1971 ; Kozlowski, 1971 ; Bonnet-Masimbert et Muller, 1973 ; Wang, 1974).

Selon Watt (1919) une dessiccation trop poussée entraîne directement la mort des glands, ces derniers ne germant plus, même après avoir repris leur teneur en eau initiale. De même pour Korstian (1927) la reprise d'eau ne constituerait pas un facteur limitant de la germination de ces semences.

Bonner (1968) a montré par contre que le péricarpe freinait la réhydratation des glands de quatre espèces de chênes nord-américains et, sur les embryons de l'une de ces espèces ( $Q$. palustris Muenchh.), que les vitesses de reprise d'eau et de germination étaient en relation étroite.

Plus récemment, l'un de nous (Lamond, 1978) a montré que l'ablation des enveloppes séminales, suivie d'un lavage de $48 \mathrm{~h}$ des embryons dénudés dans l'eau courante, stimule considérablement la germination des semences de chêne pédonculé à 
faible teneur en eau. Ces résultats nous ont incité à préciser l'influence du péricarpe sur la reprise d'eau des glands de cette espèce et, en particulier, celle des embryons à l'intérieur de leurs enveloppes.

\section{Matériel et méthode}

Cette étude a été effectuée avec des glands de chêne pédonculé (Quercus robur L.), récoltés en octobre 1976 dans la région de Lezoux (Puy de Dôme). Chaque récolte (glands d'un même arbre récoltés le même jour) a été conservée séparément pendant au moins 4 mois dans de la tourbe sèche à basse température $\left(3^{\circ} \mathrm{C}\right)$.

La première expérience a consisté à suivre la réhydratation d'une cinquantaine de semences (glands intacts et embryons nus), enfoncés aux deux tiers, micropyle vers le bas, dans de la vermiculite saturée d'eau. La reprise d'eau individuelle de ces semences a été évaluée par pesée au centigramme près, depuis la mise en germoir jusqu'à ce que la radicule de chaque germination atteigne environ $10 \mathrm{~cm}$ de longueur.

Afin de préciser l'influence des enveloppes séminales sur la reprise d'eau qui précède la germination, nous avons comparé la vitesse moyenne de réhydratation, jusqu'à l'émergence de la radicule, de lots de vingt cinq semences (glands intacts et embryons nus), provenant d'un même arbre et semées au même moment. A la fin de cette expérience, la teneur en eau des embryons inclus dans les glands intacts a été déterminée séparément.

La troisième expérience avait pour but d'analyser la perméabilité à l'eau des différents secieurs du péricarpe. Inspirée du travail de Bonner (1968), elle comportait trois lots de vingł semences :

(Témoin) - glands intacts n'ayant subi aucun traitement ;

(HB) - glands dont le hile était obturé à la paraffine ;

(EN) - embryons débarassés de leurs enveloppes séminales.

Ces semences ont été immergées dans l'eau courante et leur augmentation individuelle de poids a été évaluée après $1,2,4,7,10,15$ et 20 jours d'immersion. Cette expérience a été reprise une seconde fois avec des glands provenant d'une autre récolte.

La quatrième expérience a été réalisée afin d'étudier la reprise d'eau des embryons à l'intérieur de leurs enveloppes. Elle consistait à évaluer, à intervalles de durée croissante, la teneur en eau des embryons et celle des enveloppes séminales, de lots de vingt semences prélevées au hasard dans un ensemble de glands immergés dans l'eau courante. Réalisée une première fois avec des glands à teneur en eau relativement forte, cette expérience a été reprise avec des semences plus sèches provenant d'une autre récolte.

\section{Résultats}

\section{1. - Reprise d'eau en germoir}

Nous avons reproduit sur la figure 1 (Fig. 1) les courbes de réhydratation et d'allongement de la radicule de trois glands entiers, ayant germé plus ou moins rapidement à $24^{\circ} \mathrm{C}$.

L'on observe essentiellement que le début de croissance de la radicule est suivi 


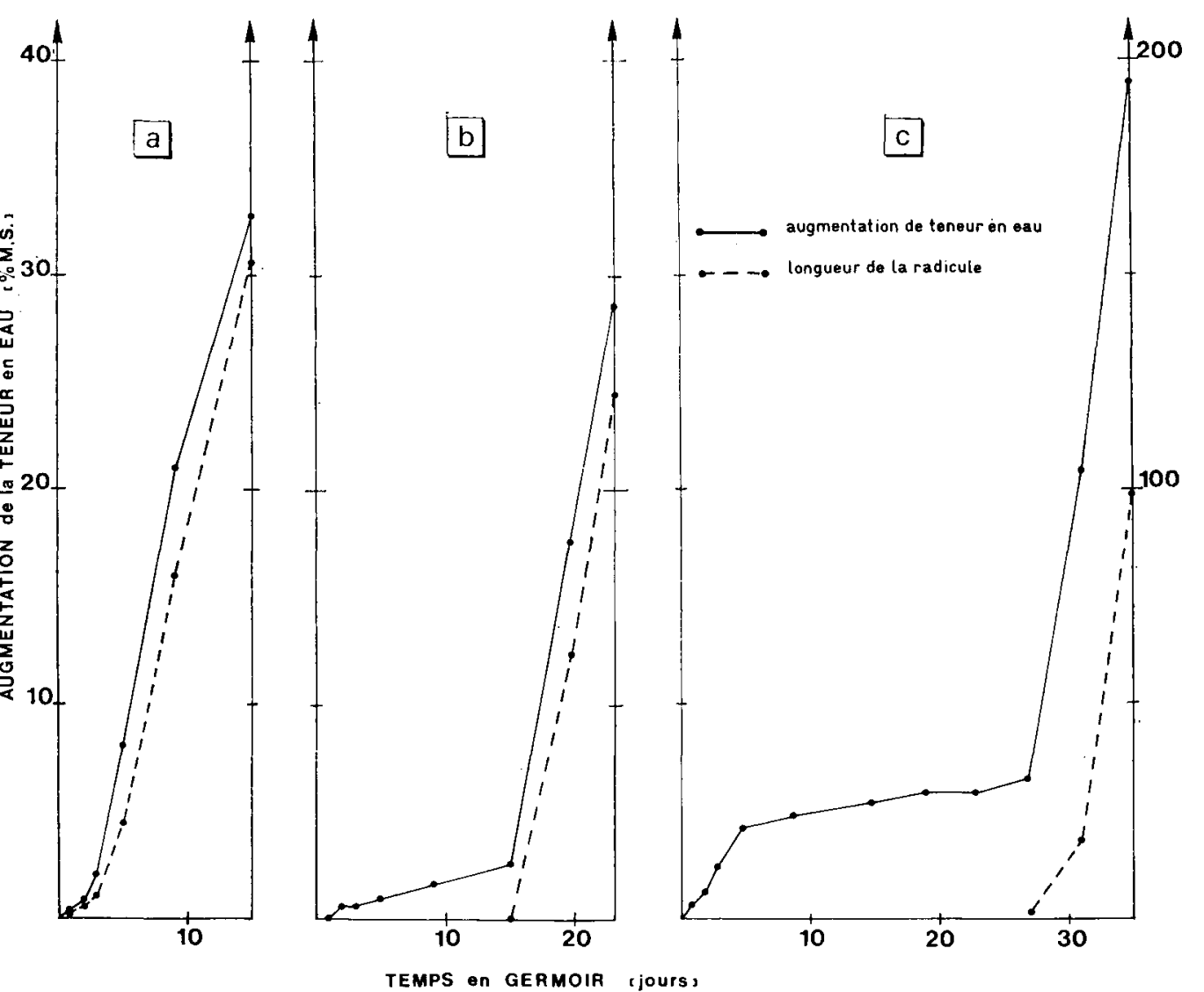

FIG. 1. - Augmentation de la teneur en eau ef allongement de la radicule de trois glands entiers mis en germoir à $24^{\circ} \mathrm{C}: a$ ) gland ayant germé très rapidement; b) individu intermédiaire ; c) gland ayant germé tardivement. Noter l'augmentation considérable de la reprise d'eau après la germination.

Increase in water content and elongation of the seminal root of three acorns planted af $24^{\circ} \mathrm{C}: a$ ) acorn who had germinated rapidly ; $b$ ) intermediate acorn ; $c$ ) acorn who had germinated lately. Note the important water uptake after germination.

d'une forte accélération de la reprise d'eau du gland et ceci, quel que soit l'intervalle de temps séparant l'ensemencement de la germination.

Avant l'émergence de la radicule, la réhydratation du gland se fait à une vitesse très réduite et, souvent, de façon graduelle; la plupart du temps on n'observe pas la phase initiale d'imbibition rapide décrite par Bonner (1968) dans le cas de glands immergés dans l'eau liquide.

La brusque accélération de la reprise d'eau consécutive à la germination semble intimement liée à la croissance de la radicule, donc à l'apparition d'une zone d'absorption au voisinage de l'extrémité de celle-ci. En effet, dans certains cas (fig. 2), bien qu'ayant percé les enveloppes séminales, la radicule ne commence pas à croître immédiatement ; l'on observe alors que la rupture de pente des courbes de réhydratation ne survient qu'au moment où cette dernière amorce sa croissance en longueur. 


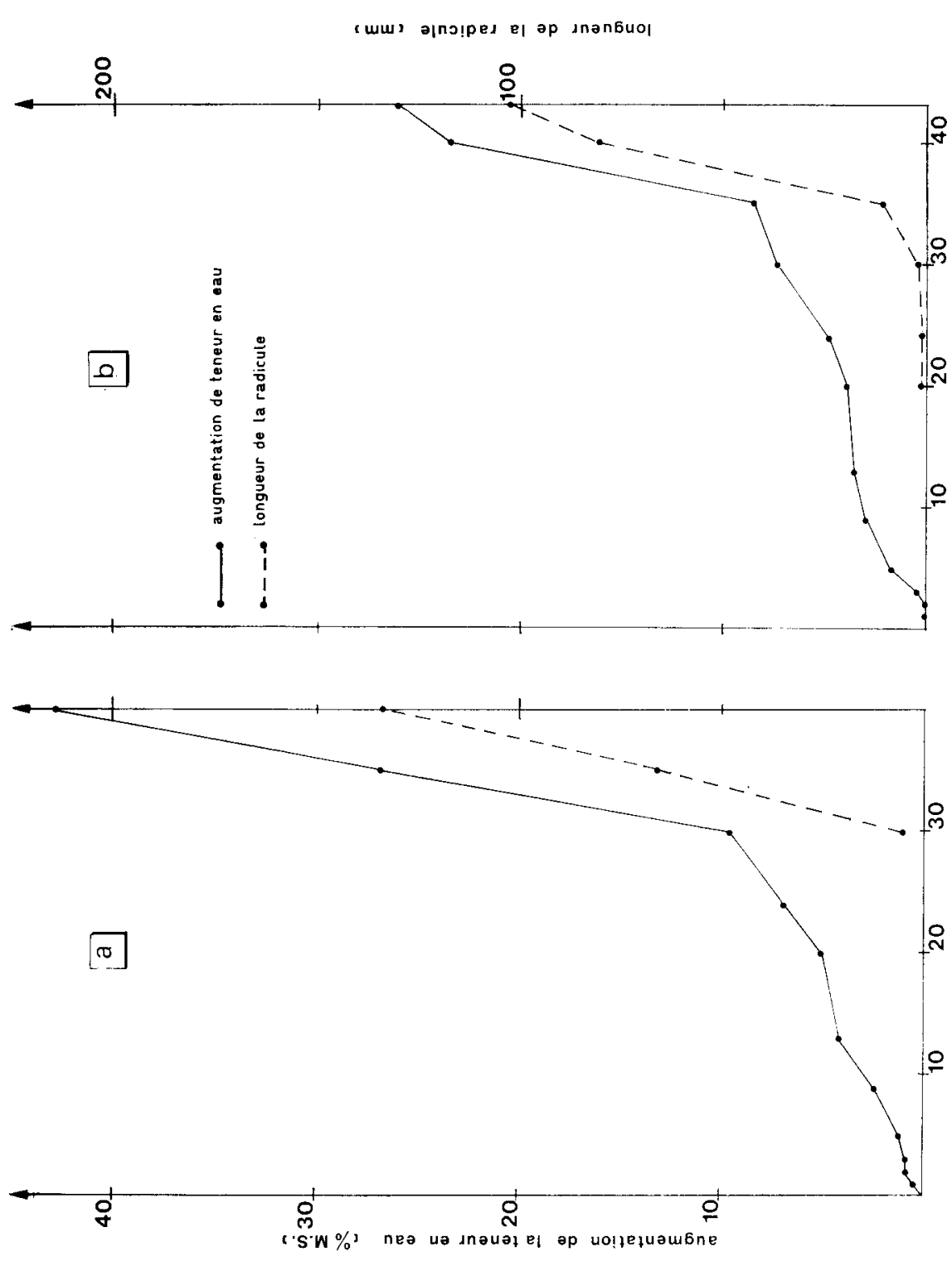

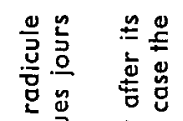

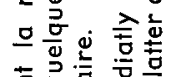

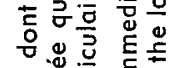

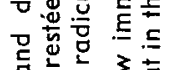
可岛过

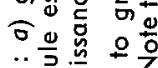

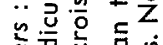

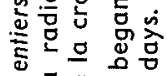

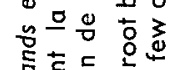

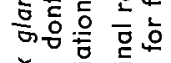

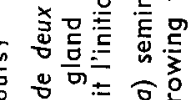

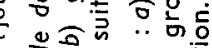

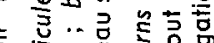

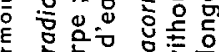
E 옹면

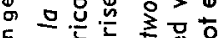
눰ㅇ⿺ㅇ

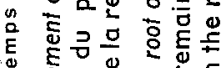

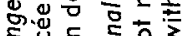
원 ᄃ 듕

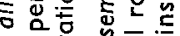

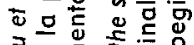

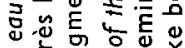

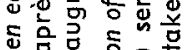

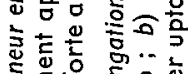

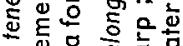

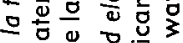
ํํㄱ온 岕口岩岳

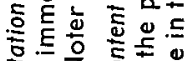

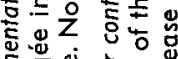

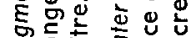
嗯은 1 는

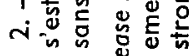
o 
Dans le cas des embryons nus, la reprise d'eau est beaucoup plus intense et la germination est très rapide (temps moyen de germination : 4 à 5 jours). L'allongement de la radicule n'entraîne pas d'augmentation marquée de la vitesse de réhydratation, même pour les embryons qui mettent le plus de temps à germer (fig. 3).

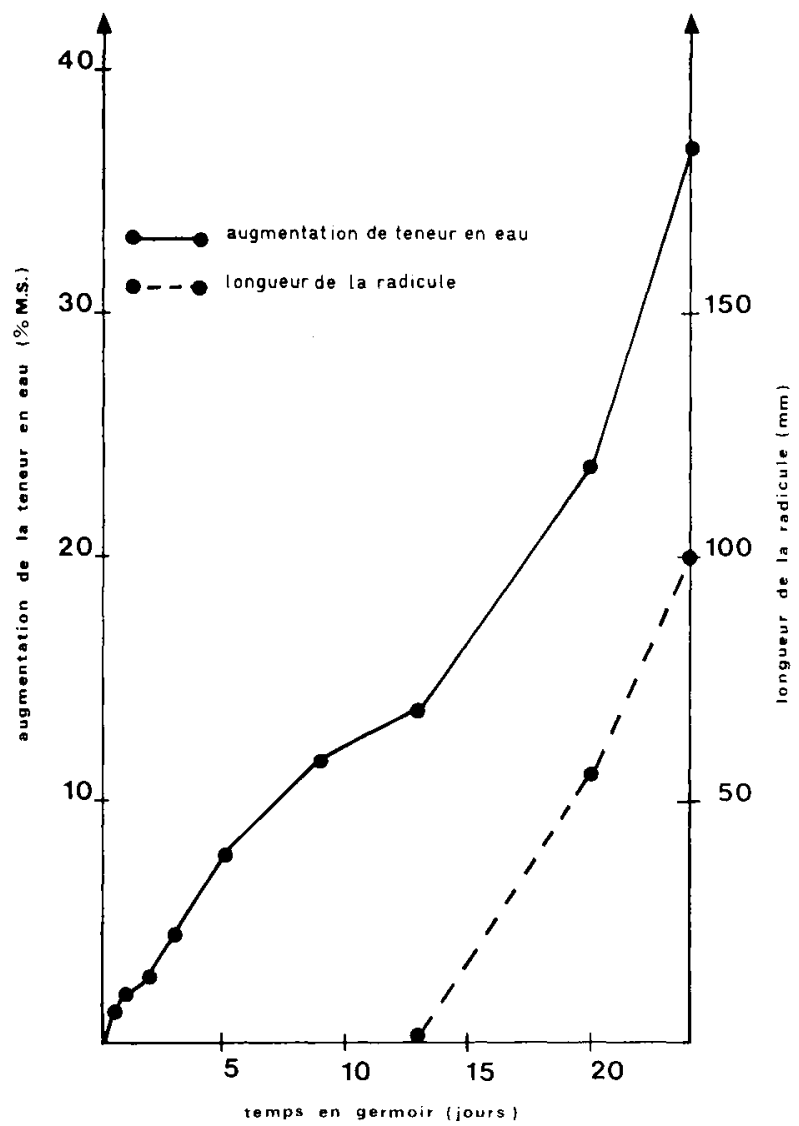

FIG. 3. - Augmentation de la feneur en eau ef allongement de la radicule d'un embryon nu ayant germé tardivement. Noter que la germination n'entraîne qu'une très faible augmentation de la vitesse de reprise d'eau.

Increase in water content and elongation of the seminal root of an isolated embryo who had germinated lately. Note that here there is only on very small increase in the water uptake after the germination.

L'absence de rupture de pente sur les courbes de réhydratation des embryons nus provient en majeure partie de la très forte reprise d'eau qui précède leur germination. Cette dernière est en effet environ quatre fois plus forte que celle des glands entiers (tabl. 1).

L'on a indiqué dans ce même tableau (tabl. 1) la teneur en eau moyenne, 1 à 2 jours après la germination, des embryons de semences mises à germer avec ou sans enveloppes séminales. Bien qu'étant restés en germoir trois fois plus longtemps que les embryons nus, les embryons extraits de leurs enveloppes séminales après la germination, ont une teneur en eau plus faible que ceux-ci. 


\section{TABLEAU 1}

Augmentation quotidienne moyenne de poids frais (p. 100 mat. sèche) ef teneur en eau moyenne des embryons (p. 100 mat. sèche) au moment de la germination, pour des lots de 25 semences enfouies aux deux tiers dans la vermiculite safurée d'eau,

Mean daily increase of the fresh weight of the seeds (p. 100 dry weight) and mean water content of the embryos (p. 100 dry weight), at the beginning of the seminal root growth, for samples of 25 seeds planted in wet terralite.

\begin{tabular}{llc} 
& Gland antier & Embryon nu \\
\hline $\begin{array}{l}\text { Augmentation journalière de poids } \\
\text { frais } \ldots \ldots \ldots \ldots \ldots \ldots \ldots \ldots \ldots \ldots \ldots\end{array}$ & $0,67 \pm 0,18 *$ & $2,58 \pm 2,34$ \\
\hline $\begin{array}{l}\text { Teneur en eau des embryons en fin } \\
\text { d'expérience } \ldots \ldots \ldots \ldots \ldots \ldots \ldots\end{array}$ & $79,8 \pm 2,38$ & $83,3 \pm 2,34$ \\
\hline
\end{tabular}

(*) Intervalle de confiance des moyennes au seuil de 5 p. 100.

(*) Confidence interval of the mean at 5 p. 100 level.

Ces résultats indiquent que la reprise d'eau des embryons, à l'intérieur de leurs enveloppes séminales, est très réduite avant la germination.

Lorsque la radicule atteint une longueur de 10 à $15 \mathrm{~cm}$, on constate par contre, une augmentation appréciable de la teneur en eau des cotylédons (tabl. 2) ; ce qui pourrait indiquer que la mobilisation des réserves cotylédonnaires est liée à une absorption d'eau importante.

\section{TABLEAU 2}

Teneur en eau moyenne des cotylédons '(p. 100 mat. sèche) de lots de 20 semences, d̀ la mise en germoir et lorsque leur pivot atteint environ 10 d̀ $15 \mathrm{~cm}$ de longueur (fin d'expérience)

Mean value in the water content (p. 100 dry weight) of the cotyledons of twenty seeds samples, at sowing and after their seminal roof had reached 10 fo $15 \mathrm{~cm}$ in length (at the end of the experiment)

Gland entier Embryon nu

\begin{tabular}{lll} 
Mise en germoir $\ldots \ldots \ldots \ldots \ldots \ldots \ldots$ & - & $72,6 \pm 4,62 *$ \\
\hline Fin d'expérience. $\ldots \ldots \ldots \ldots \ldots \ldots$ & $100,5 \pm 3,24$ & $105,4 \pm 6,52$
\end{tabular}

(*) Intervalle de confiance des moyennes au seuil de 5 p. 100 . Noter l'augmentation nettement significative de la teneur en eau de ces organes après la germination.

(*) Confidence interval of the mean at 5 p. 100 level. Note the highly significant increase in the water content of the cotyledons after the germination. 


\section{2. - Perméabilité à l'eau des enveloppes séminales}

Nous avons donné au tableau 3 (tabl. 3) l'augmentation moyenne de poids frais de glands entiers et d'embryons nus immergés pendant 20 jours dans l'eau courante.

Si l'on considère que cette augmentation de poids correspond exactement à la reprise d'eau, on note essentiellement que les embryons nus s'imbibent beaucoup plus que les glands entiers et que la réhydratation de ces derniers est encore plus faible lorsque le hile est scellé à la paraffine.

\section{TABLEAU 3}

Augmentation moyenne de poids frais (p. 100 mat. sèche) de semences immergées pendant 20 jours dans l'eau courante et leur intervalle de confiance au seuil de 5 p. 100. EN : embryons nus ; $\mathrm{Gl}$ : glands intacts ;

$\mathrm{HB}$ : glands à hile bouché. Moyenne sur 25 semences.

Mean fresh weight increase (p. 100 dry weight) of seeds immersed in running water for twenty days and confidence inferval of the means at 5 p. 100 level. EN : isolated embryos; $\mathrm{Gl}$ : whole acorns ; $\mathrm{BH}$ : acorns with paraffin sealed cup scar. Average on 25 seeds.

\begin{tabular}{llcl}
\hline Récoltes & E.N. & G.I. & H.B. \\
\hline I $\ldots \ldots \ldots \ldots$ & $38,9 \pm 3,27$ & $15,4 \pm 1,96$ & $10,9 \pm 2,02$ \\
\hline II $\ldots \ldots \ldots \ldots$ & $49,0 \pm 5,83$ & $20,0 \pm 3,58$ & $14,1 \pm 2,53$ \\
\hline
\end{tabular}

\section{TABLEAU 4}

Teneur en eau moyenne (p. 100 mat. sèche) de lots de 20 glands immergés dans l'eau couranfe pendant des temps de durée croissante ainsi que celle de leurs embryons et de leurs enveloppes séminales

Mean value water content (p. 100 dry weight) of acorns of twenty seeds samples immersed in running water for increasing period of time and the mean value in water content of their embryos and testae

\begin{tabular}{ccccccc}
\hline $\begin{array}{c}\text { Durée de } \\
\text { l'immersion } \\
\text { (jours) }\end{array}$ & \multicolumn{3}{c}{ Récolte 1 } & \multicolumn{3}{c}{ Récolte 2 } \\
\cline { 2 - 7 } & Gland entier & Embryon & Enveloppes & Gland entier & Embryon & Enveloppes \\
\hline Origine & $71,3 \pm 2,83 *$ & $78,5 \pm 3,26$ & $39,7 \pm 3,06$ & $62,1 \pm 1,88$ & $67,3 \pm 1,87$ & $38,3 \pm 4,40$ \\
1 & $77,1 \pm 3,01$ & $78,3 \pm 3,16$ & $72,8 \pm 6,90$ & $61,9 \pm 1,75$ & $62,0 \pm 1,78$ & $61,9 \pm 5,05$ \\
2 & $74,4 \pm 1,91$ & $76,1 \pm 2,15$ & $67,2 \pm 4,45$ & $66,9 \pm 1,67$ & $66,8 \pm 1,77$ & $69,7 \pm 4,67$ \\
4 & $79,4 \pm 2,85$ & $78,3 \pm 2,93$ & $85,2 \pm 5,79$ & $65,1 \pm 1,72$ & $63,6 \pm 2,15$ & $73,4 \pm 5,46$ \\
7 & $76,6 \pm 2,15$ & $74,4 \pm 2,76$ & $86,1 \pm 6,62$ & $71,4 \pm 1,53$ & $69,7 \pm 2,08$ & $79,1 \pm 7,14$ \\
10 & $83,0 \pm 3,07$ & $80,2 \pm 2,89$ & $96,1 \pm 8,46$ & $71,8 \pm 1,76$ & $69,3 \pm 1,52$ & $84,8 \pm 5,36$ \\
15 & $77,3 \pm 2,28$ & $73,8 \pm 2,43$ & $93,9 \pm 8,14$ & $74,3 \pm 1,73$ & $71,5 \pm 2,08$ & $87,4 \pm 5,50$ \\
20 & $83,5 \pm 3,67$ & $80,7 \pm 3,85$ & $95,6 \pm 5,58$ & $70,8 \pm 1,68$ & $67,6 \pm 2,68$ & $89,9 \pm 3,99$
\end{tabular}

(*) Intervalle de confiance des moyennes au seuil de 5 p. 100 . Noter la similitude des teneurs en eau des embryons de différents lots de glands appartenant à une même récolte.

(*) Confidence interval of the mean at 5 p. 100 level. Note that the water content of the embryos is similar in all the samples of the same crop. 
Autrement dit, les enveloppes séminales réduisent considérablement la réhydratation du gland ef le hile constitue la zone la plus perméable du péricarpe.

L'étude de la cinétique de réhydratation de ces différents lots de semences (fig. 4) montre en outre que la vitesse de reprise d'eau des embryons isolés diminue de façon progressive durant toute la durée de l'expérience tandis que celle des glands entiers chute brusquement après le deuxième jour d'immersion. Les glands à hile scellé à la paraffine reprennent environ trois fois moins d'eau que les témoins durant les premières $48 \mathrm{~h}$; leur teneur en eau tend ensuite à rejoindre celle de ces derniers.

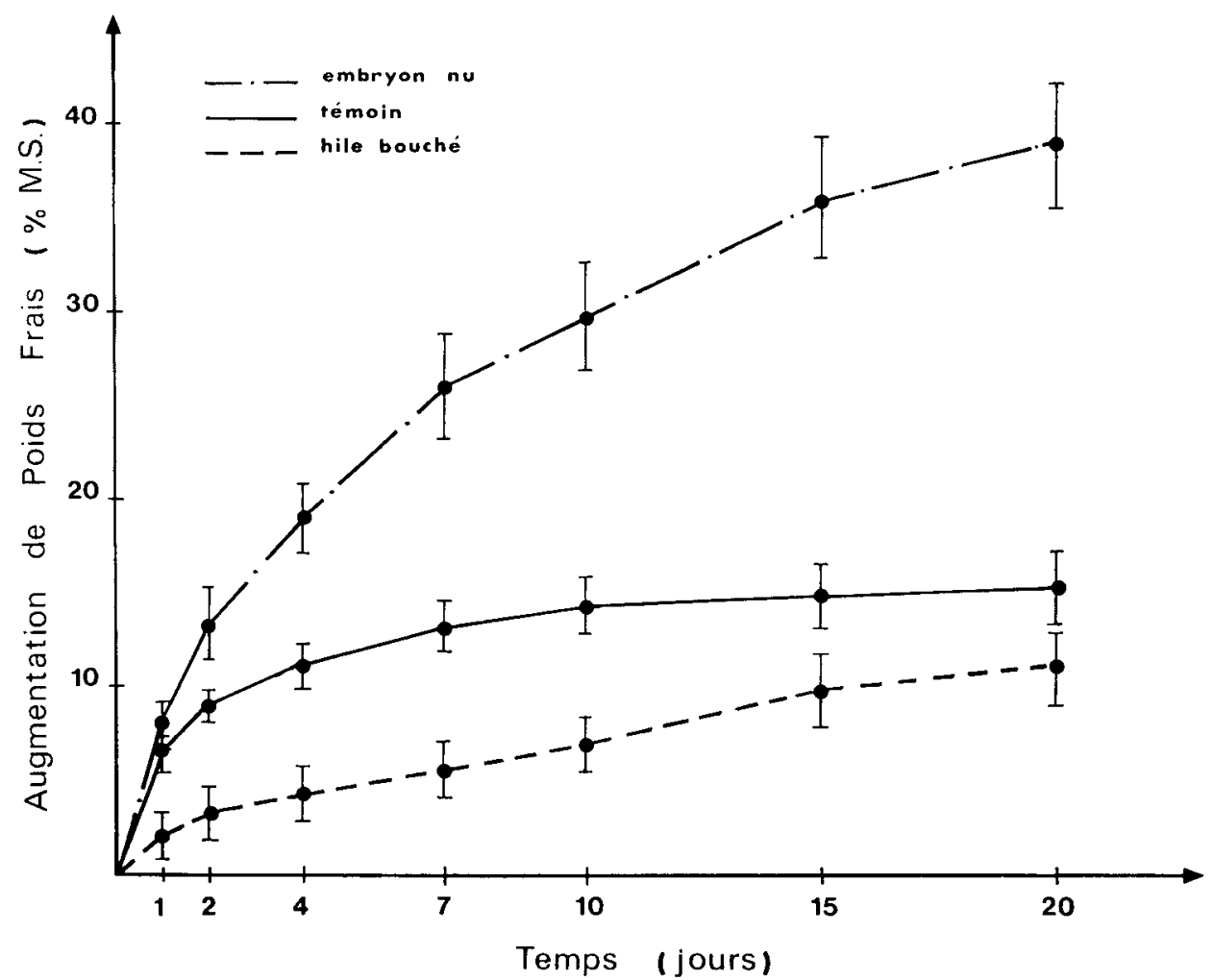

FIG. 4. - Augmentation moyenne de poids frais (p. 100 mat. sèche) en fonction du temps, de lots de vingtcinq semences immergées dans l'eau courante. Les traits verticaux représentent l'intervalle de confiance des moyennes au seuil de 5 p. 100.

Mean increase in fresh weight (p. 100 dry weight) as a function of the time for twenty five seeds samples, soaked in running water. Vertical bars represent confidence interval at 5 p. 100 level.

\section{3. - Réhydratation des embryons dans leurs enveloppes}

La comparaison de la teneur en eau moyenne des embryons extraits de lois de glands ayant séjourné durant des temps croissants dans l'eau courante (tabl. 4) montre que ces derniers ne reprennent pas d'eau de façon appréciable à l'intérieur de leurs enveloppes, tout au moins pendant les 20 premiers jours; ceci quelle que soit leur teneur en eau au moment de l'immersion. 
La teneur en eau des enveloppes séminales augmente par contre très fortement au cours des premiers jours d'immersion.

L'accroissement de la teneur en eau des glands entiers est donc du quasi uniquement à la réhydratation de leurs enveloppes séminales.

\section{Discussion}

La reprise d'eau en germoir des glands de chêne pédonculé présente deux phases bien distinctes : une phase d'imbibition lente et graduelle, suivie d'une phase de réhydratation beaucoup plus rapide, commençant avec le début de la croissance de la radicule.

En règle générale, la reprise d'eau qui précède la germination ne se subdivise pas en phase initiale d'imbibition rapide et phase « plateau », comme dans le cas des courbes fournies par de nombreux auteurs (Stone, 1958 ; Hubac, 1961 ; Côme, 1967 ; Verdier, 1975 ; ...) pour des semences d'espèces variées.

L'absence de phase initiale d'imbibition rapide résulte sans aucun doute de la très faible perméabilité des secteurs du péricarpe en contact avec le substrat humide. En effet, lorsque les glands sont immergés dans l'eau liquide, cette phase est beaucoup moins nette lorsque le hile a été préalablement scellé à la paraffine (fig. 4).

Le rôle négligeable du micropyle dans la reprise d'eau des glands de chêne pédonculé avaił déjà été signalé par Watt (1919). Korstian (1927) et plus récemment Bonner (1968) ont montré en outre que les enveloppes séminales freinent considérablement la reprise d'eau des glands et que le hile constitue la zone la plus perméable du péricarpe.

Les mesures de reprise d'eau que nous avons effectuées sur des glands et des embryons nus, tant en germoir que dans l'eau liquide, confirment pleinement les observations de ces auteurs. Elles indiquent de plus que la réhydratation des embryons à l'intérieur des enveloppes séminales est extrêmement faible jusqu'à la germination.

Nous ignorons encore les causes de la mauvaise réhydratation des embryons à l'intérieur de leurs enveloppes. II ne s'agit vraisemblablement pas d'une incapacité de l'embryon lui-même, puisque celui-ci se réhydrate parfaitement lorsqu'il est directement en contact avec de l'eau liquide. La faible perméabilité des enveloppes ne semble pas non plus constituer une explication suffisante puisque l'embryon ne reprend pas d'eau même lorsque ces dernières sont bien imbibées.

Des mesures récentes montrent que la réhydratation de l'embryon de chêne pédonculé s'accompagne d'une augmentation de volume correspondant à celui de l'eau absorbée, tandis que le volume de glands intacts, placés dans les mêmes conditions, varie très peu. Ces résultats semblent indiquer que la faible reprise d'eau des embryons à l'intérieur de leurs enveloppes pourrait provenir d'une contrainte mécanique exercée par le péricarpe, analogue à celle observée par Istanbouli (1974) sur les semences d'olivier.

La reprise d'eau importante des embryons à l'intérieur de glands aux enveloppes trouées par endroits, ainsi que l'augmentation proportionnelle du volume de ces semences, suivie après quelques jours d'imbibition de l'éclatement du péricarpe au niveau du micropyle, montrent cependant que le péricarpe esł doué d'une certaine élasticité et que les forces développées par la réhydratation de l'embryon peuvent provoquer sa rupture. 
Ce dernier résultat confirme que, quelle qu'en soit la cause, la reprise d'eau de l'embryon de chêne.pédonculé ne peuł s'effectuer que lorsque la surface de ce dernier entre en contact partiel ou total avec le substrat humide. Cet effet nettement dépressif des enveloppes sur la réhydratation des embryons constitue sans aucun doute l'une des principales causes de la mauvaise germination des glands à teneur en eau relativement faible (30-35 p. 100 mat. fraîche). Rappelons à ce propos, que Bonner (1968) a montré que les vitesses de germination et de réhydratation d'embryons nus de Quercus palustris Muenchh. immergés dans des solutions de saccharose de concentration croissante, étaient en relation étroite avec la pression osmotique de ces dernières.

Dans un travail ultérieur, l'un de nous (Levert 1977) a observé l'existence d'une étroite corrélation entre la teneur en eau de glands entiers à la mise en germoir et leur vitesse de germination. Ce résultat renforce l'hypothèse d'un lien étroit entre l'énergie de germination et les disponibilités hydriques de l'embryon.

Selon Steinmetz (1970) ef Rohmeder (1977), le seuil critique de déshydratation des glands des chênes européens se situe entre 25 ef 35 p. 100 d'eau par rapport à la matière fraîche. Holmes et Buszewicz (1956) ainsi que Suszka (1976) précisent en outre qu'une bonne conservation des glands de chêne pédonculé nécessite une teneur en eau de 40 d̀ 45 p. 100.

Ce seuil critique de déshydratation, relativement élevé par rapport à celui de plusieurs autres semences forestières, résulte peut-être en partie de l'incapacité des embryons de chêne à se réhydrater de façon appréciable à l'intérieur de leurs enveloppes. En effet, les travaux réalisés jusqu'à maintenant dans ce domaine sont basés sur des tests de germination de glands entiers, à l'intérieur desquels les embryons ne reprennent pratiquement pas d'eau. Dans cette hypothèse, il devrait être possible de conserver à très basse température des glands beaucoup plus secs, dans la mesure où l'ensemencement serait précédé d'une réhydratation convenable des embryons après ablation partielle ou totale des enveloppes.

\section{Conclusion}

Les glands intacts de chêne pédonculé reprennent peu d'eau avant la germination, et seules les enveloppes se réhydratent pendant cette période. En effet, le péricarpe est peu perméable sauf au niveau du hile ef il inhibe presque totalement la reprise d'eau de l'embryon lui-même.

Cet effet très dépressif du péricarpe sur l'imbibition de l'embryon est sans doute à l'origine de la mauvaise germination des glands à teneur en eau inférieure à 35 p.100. Cette difficulté peut cependant être surmontée par une réhydratation convenable des embryons après ablation tolale ou partielle des enveloppes séminales.

L'ablation des enveloppes séminales, facile à réaliser manuellement, ne pose aucun problème à l'échelle du laboratoire. Vue à l'échelle forestière, cette opération nécessite la mise au point préalable, qui pose vraisemblablement des problèmes techniques, d'un procédé mécanique de décortication des glands.

Reçu pour publication en avril 1979. 


\section{Remerciements}

Cette étude a été rendue possible grâce à l'aide financière de la DGRST (Contrat no 77.7.11.35). financière.

L'auteur principal, boursier du Gouvernement français, tient à remercier celui-ci pour son aide

\section{Summary}

\section{Influence of the pericarp on the inhibition of quercus robur acorns}

Water uptake of planted whole acorns of Quercus robur L. is very slow and progressive until germination occurs. On the other hand, isolated embryos of this seed steadily absorb a high amount of water when they are embedded in a moist substratum.

Experiments performed with seeds (isolated embryos, whole acorns, acorns with paraffin sealed cup scar) immersed in running water have shown :1) that the cup scar is the most permeable area of the pericarp ; 2) that the water uptake of embryos is very poor in the acorn even when the water content of the pericarp is very high.

The low permeability of those parts of the pericarp generally embedded in the soil and the negligible water uptake of the embryo itself, inside the acorn, are probably responsible, in a large part, for the poor germination of relatively dry acorns.

\section{Références bibliographiques}

BONNER F. T., 1968. Water uptake and germination of red oak acorns. Bot. Gaz., 129 (1), $83-85$.

BONNER F. T., 1971. Storage of acorns and other large hardwood seeds. Problems and possibilities. Proceedings Southeastern nurserymen's conferences, Southeastern Area, State \& private forestry, Atlanta, Ga., 77-82.

BONNET-MASIMBERT M., MULLER C., 1973. La conservation des faines et des glands, recherches et perspectives. Bulletin technique Office Notional des Forêts, 5, 13-19.

BONNET-MASIMBERT M., MULLER C., MORELET M., 1977. De nouveaux espoirs pour la conservation des glands. Bullefin Technique Office National des Forêts, 9, 47-54.

CAMUS A., 1952-1954. Monographie du Genre Quercus. Paul Lechevalier, Paris.

CLAUDOT M., 1974. Indications pour la compagne 1974-75 de récolte, de conservation ef de semis de glands. CTGREF, groupement d'Aix en Provence, division P. F. C. I. Mémoire no 2, 31 p.

CÔME D., 1967. L'inhibition de germination des graines de pommier (Pirus malus L.) non dormantes. Rôle possible des phénols téguınentaires. Ann. Sc. Nat. Bot., 8, 371-478.

HOLMES G. D., BUSZEWICZ G., 1956. Longevity of acorns with several storage methods. Rep. Forest. Res., 88-99.

HUBAC C., 1961. Recherches sur l'évolution de l'aptitude à l'anhydrobiose au cours de la vie embryonnaire ef de la germination. Thèse $3^{\mathrm{e}}$ Cycle, Paris, $135 \mathrm{p}$.

ISTANBOULI A., 1974. Etude de la «dormance » des semences d'olivier (Olea europoea L.). Rôle des enveloppes dans l'imbibition de la graine ef de l'embryon. Rev. gén. Bot., 81, 215-21.

KORSTIAN, 1927. Factors controlling germination and early survival in oaks. Yale Univ. Sch. Forest. Bull., 19, 115 p.

KOZLOWSKI T. T., 1971. Growth and development of trees. Vol. 1, Seed Germination, Ontogeny and shoof growth. Academic Press, New York, 443 p.

LAMOND M., 1978. Péricarpe et cinétique de germination des glands de chêne pédonculé. Ann. Sci. forest., 35, 203-210.

LEVERT J., 1977. Etude de l'influence de quelques facteurs physiques sur la germination des glands de chêne pédonculè (Q. robur L.). D. E. A., Université de Clermont II, 51 p.

ROHMEDER E., 1977. Dar Saatgut in der Forestwirlschoft. Ed. Paul Parey, Hambourg.

STEINMETZ G., 1970. Expérience de conservation des glands de chêne pédonculé. C.T.G.R.E.F., Nogentsur-Vernisson, rapport 1969-1970.

STONE E. C., 1958. The seed dormancy mechanism in Pine. Physiology of Forest Trees. Ed. K. V. Thimann, Ronald Press London, 611-628.

SUSZKA B., 1976. Studies on the long-ferm storage of acorns. Polish Acad. of Sc., Inst. of Dendrology Kornik, $6 \mathrm{e}$ rapport annuel, $112 \mathrm{p}$.

VERDIER P., 1975. Recherches sur la germination du Maïs. Thèse $3^{\mathrm{e}}$ Cycle, Clermont-Ferrand, 172 p.

WANG B. S. P., 1974. Tree seed storage. Department of the environment, Canadian Forestry Service, Publication no 1335,32 p., Ottawa 1974.

WATT A. S., 1919. On the causes of failure of natural regeneration in british oakwoods, J. Ecol., 7, 173-203. 